\title{
Second-Layer Induced Island Morphologies in Thin-Film Growth of Fullerenes
}

\author{
Martin Körner, ${ }^{1}$ Felix Loske, ${ }^{1,2}$ Mario Einax, ${ }^{1}$ Angelika Kühnle, ${ }^{2}$ Michael Reichling, ${ }^{1}$ and Philipp Maass ${ }^{1, *}$ \\ ${ }^{1}$ Fachbereich Physik, Universität Osnabrück, Barbarastraße 7, 49076 Osnabrück, Germany \\ ${ }^{2}$ Institut für Physikalische Chemie, Johannes Gutenberg-Universität Mainz, 55099 Mainz, Germany
}

(Received 25 March 2011; published 29 June 2011)

\begin{abstract}
Deposition of fullerenes on the $\mathrm{CaF}_{2}(111)$ surface yields peculiar island morphologies with close similarities to previous findings for (100) surfaces of other ionic crystals. By means of noncontact atomic force microscopy we find a smooth transition from compact, triangular islands to branched hexagonal islands upon lowering the temperature. While triangular islands are two monolayers high, hexagonal islands have a base of one monolayer and exhibit a complicated structure with a second-layer outer rim and trenches oriented towards the interior. By developing a kinetic growth model we unravel the microscopic mechanisms of the structure formation.
\end{abstract}

PACS numbers: 68.55.A-, 68.55.J-, 81.15.Aa

Tailoring of organic nanostructures on insulating substrates is currently considered to be a major challenge for molecular electronics [1,2]. In related self-assembled growth processes [3-5], it is of particular relevance to understand which types of island morphologies develop on a substrate and how they can be controlled. Related questions have been extensively studied in the context of the growth of thin metal films and semiconductor nanostructures [6]. A large variety of island morphologies has been found in these material systems and a common feature of them is that they are, in addition to the deposition flux and growth temperature, strongly influenced by properties related to the substrate, as, for example, lattice mismatch, edge and corner diffusion as well as substrateadsorbate interactions [6-8].

For the growth of fullerenes on insulating substrates, the comparably weak molecule-substrate interaction results in high molecular diffusivity and a process that has been described as molecular dewetting [9]. It has been speculated that a competition between commensurate and coincident molecular structures governs both dewetting and the resulting island shapes. So far, however, the complex island morphologies formed upon dewetting have not been understood.

In this Letter we demonstrate that for thin-film growth of fullerenes on an insulating surface the second-layer occupation is the decisive factor that determines the island shapes. Effectively, the morphology is generated from the top rather than the bottom of the fullerene islands. This qualitative difference compared to island growth on metallic substrates originates from the weak moleculesubstrate interaction for such a system and is exemplified here for the self-assembled growth of $\mathrm{C}_{60}$ on $\mathrm{CaF}_{2}(111)$. By means of noncontact atomic force microscopy (NC-AFM) we reveal different types of morphologies as a function of substrate temperature. By developing a kinetic growth model we are able to uncover the underlying growth mechanisms and to reproduce the morphologies by kinetic Monte Carlo (KMC) simulations.
Experiments are performed in an ultrahigh vacuum system allowing the in situ preparation of large atomically flat (111) oriented terraces on $\mathrm{CaF}_{2}$ by cleavage [10]. $\mathrm{C}_{60}$ molecules are deposited at a flux of $F=5 \times 10^{-4} \mathrm{~s}^{-1} \mathrm{~nm}^{-2}$ for $2 \mathrm{~min}$ with the substrate kept at temperatures of 319,308 , and $297 \mathrm{~K}$. NC-AFM measurements are performed after cooling the sample to room temperature with equipment and procedures as described earlier [11]. Upon deposition at $319 \mathrm{~K}$, islands are compact triangles with truncated vertices as shown in Fig. 1(a). The exemplary island shown in Fig. 1(d) with a corresponding line scan in $(\mathrm{g})$ demonstrates that islands
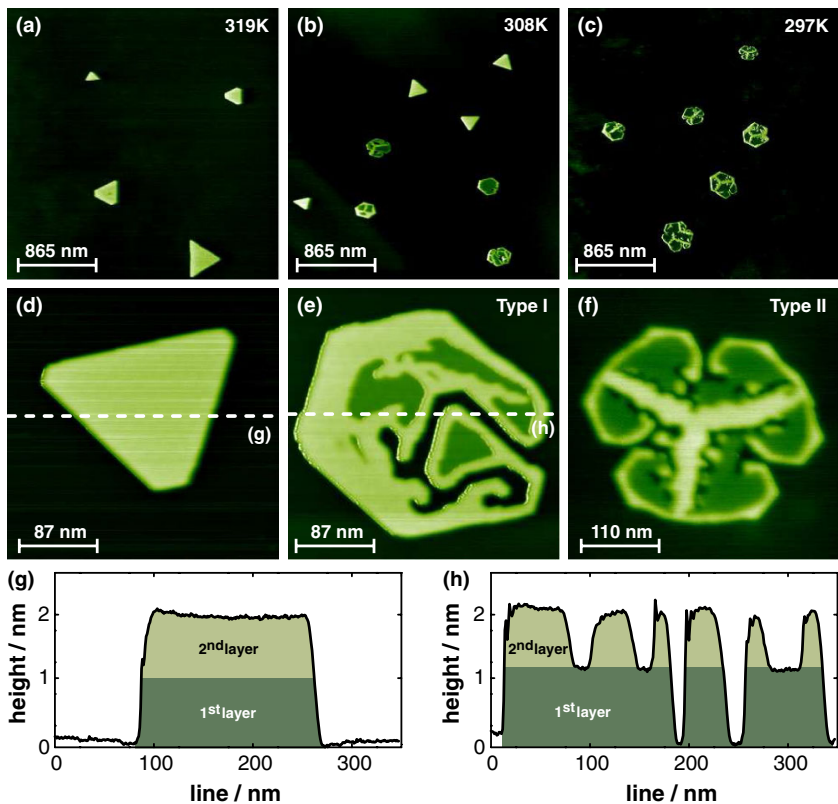

Type I
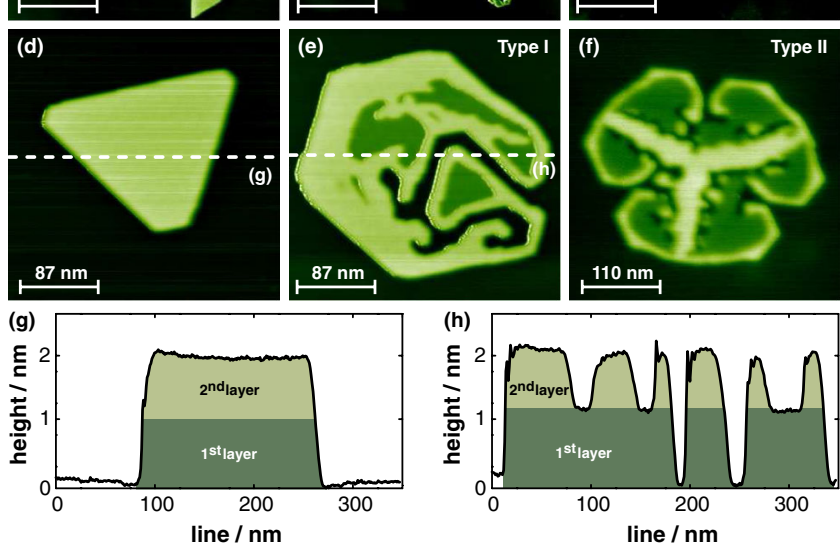

FIG. 1 (color online). (a)-(c) NC-AFM images of $\mathrm{C}_{60}$ islands on $\mathrm{CaF}_{2}(111)$ at three different growth temperatures. (d)-(f) Magnified images of single islands: a compact triangle (d), and hexagonal islands with morphologies I (e) and II (f). (g), (h) Height profiles along lines scans shown in (d),(e). 
always consist of two complete $\mathrm{C}_{60}$ layers, a feature found even at very low coverages. Upon deposition at $297 \mathrm{~K}$, the islands have an overall hexagonal outer shape [Fig. 1(c)] with a branched inner structure formed by trenches oriented towards the islands' interior. Two exemplary islands are shown in Figs. 1(e) and 1(f). Line scans as displayed in (h) reveal that these islands have a one-layer base and a second-layer rim. Taking a closer look at the branched islands, two subtypes can be distinguished. Type I islands [Fig. 1(e)] have no inner framework, whereas type II islands [Fig. 1(f)] exhibit a dendritic second-layer structure with three fingers. Between 297 and $319 \mathrm{~K}$, there is a regime of coexisting triangular and hexagonal islands. At $308 \mathrm{~K}$ the fractions of both types are approximately equal [Fig. 1(b)]. The uncommon hexagonal structures presented here resemble the islands that have previously been observed upon dewetting of $\mathrm{C}_{60}$ on $\operatorname{KBr}(100)$ and $\mathrm{NaCl}(100)$ [12].

To model the growth of $\mathrm{C}_{60}$ clusters on $\mathrm{CaF}_{2}(111)$ we consider two layers, the first one referring to the $\mathrm{C}_{60}$ molecules on the $\mathrm{CaF}_{2}$ substrate and the second one to the $\mathrm{C}_{60}$ on top of $\mathrm{C}_{60}$ formed in the first layer. The $\mathrm{C}_{60}$ molecules are deposited with a flux $F$ and, if landing on the substrate, they diffuse until attaching to an already existing $\mathrm{C}_{60}$ island or another single $\mathrm{C}_{60}$ molecule. In view of the large $\mathrm{C}_{60}$ diameter $(\sim 1 \mathrm{~nm})$ compared to the lattice constant of the $\mathrm{CaF}_{2}(111)$ surface $(386 \mathrm{pm})$, it is convenient to use a triangular lattice with a spacing matching the $\mathrm{C}_{60}-\mathrm{C}_{60}$ bond distance in the monolayer, and to model the diffusion by effective jumps between nearest neighbor sites of this lattice with a rate $D_{1}=\nu_{1} \exp \left(-E_{1} / k_{B} T\right)$ [13]. Here $\nu_{1}$ is an attempt frequency and $E_{1}$ the activation energy for $\mathrm{C}_{60}$ diffusion on the $\mathrm{CaF}_{2}$ substrate. Interactions between neighboring $\mathrm{C}_{60}$ are taken into account by a bond energy $E_{b}$, which enters the barrier for $\mathrm{C}_{60}$ jumps along steps as an additional contribution $\left(z-z^{\prime}\right) E_{b} / 2$ to $E_{1}$, where $z$ and $z^{\prime}$ are the in-plane coordination numbers of the $\mathrm{C}_{60}$ at the initial and target site, respectively.

$\mathrm{C}_{60}$ molecules landing on the first-layer diffuse by jumps between neighboring sites of a hexagonal lattice formed by the already condensed $\mathrm{C}_{60}$ molecules with a rate $D_{2}=$ $\nu_{2} \exp \left(-E_{2} / k_{B} T\right)$. The hexagonal lattice is composed of two equivalent triangular sublattices and a cluster of $\mathrm{C}_{60}$ molecules can occupy the sites of either one of these two sublattices. Again an additional contribution $\left(z-z^{\prime}\right) E_{b} / 2$ is added to the bare activation energy $E_{2}$ for $\mathrm{C}_{60}$ at steps in the second layer. Because of their contact to three further $\mathrm{C}_{60}$ molecules underneath and the Ehrlich-Schwoebel barrier at step edges $[14,15]$, we ignore downward transitions of $\mathrm{C}_{60}$ molecules from the second to the first layer.

The weak interaction with the $\mathrm{CaF}_{2}$ surface on the other hand enables upward transitions of $\mathrm{C}_{60}$ molecules from the first to the second layer with a rate $D_{12}=$ $\nu_{12} \exp \left(-\Delta E_{12} / k_{B} T\right)$. An important aspect of our modeling is that such dewetting transitions are facilitated by $\mathrm{C}_{60}$ molecules close to step edges in the second layer, since these can help a $\mathrm{C}_{60}$ to be better coordinated during an upward transition. To include this effect, we set $\Delta E_{12}=E_{12}+\left(z-z^{\prime}\right) E_{b} / 2$, where $E_{12}$ is the bare dewetting barrier, and $z$ and $z^{\prime}$ are again the in-plane coordinations at the initial and target sites, respectively. Only in-plane bonds are counted since all other contributions to the dewetting barrier can be considered as independent of the local configuration and thus be included into $E_{12}$. In the following we will refer to upward jumps with $z^{\prime} \geq 1$ as "facilitated dewetting" transitions.

Estimates for the parameters are taken from the available literature. Recent experiments yield $E_{1} \simeq 0.214 \mathrm{eV}$ and $\nu_{1} \simeq 10^{12} \mathrm{~s}^{-1}$ for substrate diffusion of $\mathrm{C}_{60}$ on $\mathrm{CaF}_{2}(111)$ [11]. Pair potential calculations [16] and molecular dynamics simulations [17] provide values for $E_{2} \simeq 0.178 \mathrm{eV}$ and $\nu_{2} \simeq 0.2 \times 10^{12} \mathrm{~s}^{-1}$. Electronic structure calculations [18] yield an estimate of $E_{b}=0.271 \mathrm{eV}$ for the $\mathrm{C}_{60}$ bond energy (see also [16]). Setting $\nu_{12}=\nu_{1}$ we are then left with one unknown parameter, namely $E_{12}$ characterizing the dewetting barrier. As shown below, good agreement with the experimental observations is obtained for $E_{12}=0.42 \mathrm{eV}$. For quick reference, we have summarized the parameters in [19]. KMC simulations were performed using a continuous-time algorithm as described earlier [20].

Figure 2 shows simulated islands of the model for a substrate area of $1 \mu \mathrm{m}^{2}$ and the same flux as used in the experiments. In stunning similarity to the experiments shown in Fig. 1, the simulation yields double-layer truncated triangles at high temperature, while at lower temperature, hexagons with the characteristic rims and trenches develop. There is also an intermediate temperature range with a coexistence of both, triangles and hexagons. We can tune this temperature to agree with the experimentally observed value of $T \simeq 308 \mathrm{~K}$ by choosing $E_{12}=0.42 \mathrm{eV}$ for the dewetting energy.

Next we discuss the mechanisms behind the formation of the peculiar island shapes and start with the triangles.
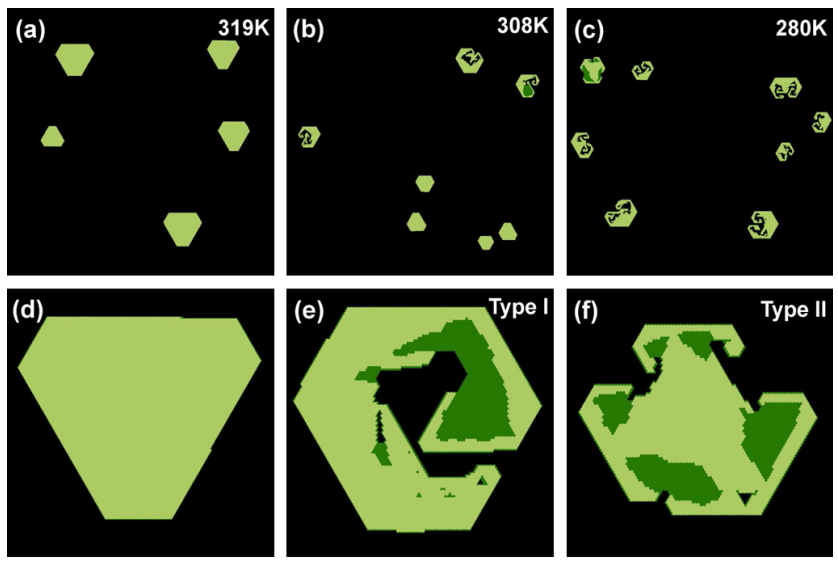

FIG. 2 (color online). (a)-(c) Simulated configurations of the growth model at different temperatures. (d)-(f) Magnified single island structures from the simulations resemble the same morphologies as found in experiments [Figs. 1(d)-1(f)]. 
At high temperature, the dewetting barrier can be surmounted by $\mathrm{C}_{60}$ molecules on a time scale smaller than or comparable to the mean interval needed for a newly deposited $\mathrm{C}_{60}$ to attach to an island. This causes islands to become occupied in the second layer already at a stage when they are composed of just a few molecules. During further growth, the $\mathrm{C}_{60}$ in the second layer facilitate upward transitions of $\mathrm{C}_{60}$ attaching to island edges and accordingly a double-layer structure is maintained.

Despite the isotropic diffusion flux of $\mathrm{C}_{60}$ molecules from the substrate areas surrounding the islands, island growth is anisotropic. This anisotropy stems from the two types of step edges of the double-layer islands, denoted as $A$ and $B$ in Fig. 3. Starting from an initially hexagonal island, further attaching $\mathrm{C}_{60}$ molecules diffuse along the island edges as long as there is no free site in the second layer. However, if two $\mathrm{C}_{60}$ molecules meet at an $A$-type step or at a corner of an $A$ - and $B$-type step, a vacant second-layer site is created and upward transitions become possible (see Fig. 3). After an upward transition of a $\mathrm{C}_{60}$, the other $\mathrm{C}_{60}$ molecule is immobilized and the island has grown in a direction perpendicular to the $A$ step. In contrast, a corresponding process at a $B$-type step requires three molecules to meet and is, therefore, less likely. Eventually the favorable growth perpendicular to $A$ steps leads to triangular islands with $B$ steps. The mechanism has some resemblance to the asymmetric corner diffusion found earlier in metal epitaxy, where an effectively higher flux around the corner in the direction of one edge leads to a favored growth of the other edge [21,22]. An essential point here is that the growth anisotropy is due to the second-layer occupation, since otherwise $A$-type steps could not be distinguished from $B$ type steps. Because of the increasing size ratio of $B$ to $A$ steps, a balance is finally reached between the total numbers of elementary upward jump processes at $B$ and $A$ steps. This causes the triangles to be truncated at their corners, as seen in Figs. 1(d) and 2(d).

At low temperatures, where the typical attachment time is smaller than the typical dewetting time, the islands do not grow as double layers. There is no anisotropy and the overall island shapes are hexagonal. For the inner structure

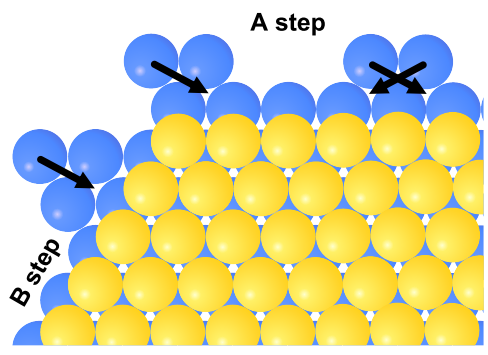

FIG. 3 (color online). Sketch of elementary upward jump processes facilitating the growth of a double-layer triangle. The processes at the $A$ step and the corner between the $A$ and $B$ step involve only two $\mathrm{C}_{60}$ and are, therefore, more likely than the process at the $B$ step involving three $\mathrm{C}_{60}$ molecules.

and the formation of two different morphologies I and II, the second layer again plays the key role. The dominating process for second-layer occupation is now the deposition on top of islands. Rare events of upward jumps overcoming the bare dewetting barrier can also generate $\mathrm{C}_{60}$ in the second layer but these become increasingly unlikely with lower temperature. Once a stable cluster has nucleated on top of an island, it will typically capture all further atoms deposited on this island, since nucleation of further secondlayer islands is unlikely in the presence of an already existing one. The origin of the morphologies I and II can be traced back to different locations of stable nucleus formation, as demonstrated in Fig. 4. This figure shows simulation results for island evolution with morphologies I [upper row (a)-(d)] and II [lower row (e)-(h)], where the $\mathrm{C}_{60}$ deposition is stopped after frames (b) and (f), respectively. In the postdeposition regime, the formation of second-layer structures from first-layer material results in a decrease of the totally covered surface area for islands of both morphologies.

A hexagonal island of type I develops, if the stable nucleus in the second layer forms at or close to the island edge, so that the growth of the nucleus leads to an early contact with a step edge [Fig. 4(a)]. At the moment of contact, the $\mathrm{C}_{60}$ in the second layer can facilitate upward jumps and the newly arriving $\mathrm{C}_{60}$ in the second layer in turn facilitate further dewetting transitions. As a consequence, a rim is formed along the island edge [Fig. 4(b)]. During deposition, the island area extends faster by newly attaching $\mathrm{C}_{60}$ to the rim-free edges than to the edges with rim, where facilitated dewetting leads to a broadening of the rim. The faster extension of the island area at rim-free edges makes it unlikely for the growing rim to surround the island during deposition. In the postdeposition regime, however, the rim further grows by $\mathrm{C}_{60}$ molecules that diffuse along the island edges, reach one of the two rim ends and extend it by a facilitated upward transition. These $\mathrm{C}_{60}$ molecules stem from the rim-free edges of the island. As long as the two rim ends are located at different island edges, the rim-free edges can remain straight on average. However, when the two rim ends approach
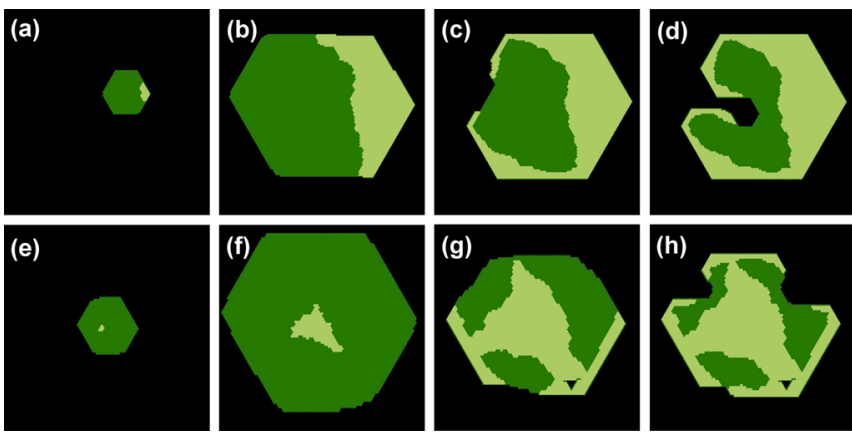

FIG. 4 (color online). Hexagonal islands from the KMC simulations showing the evolution of the two morphologies I [upper row (a)-(d)] and II [lower row (e)-(h)]. 
each other along the same edge, a funnel starts to form [Fig. 4(c)]. Further growth of this funnel due to the continued buildup of the rim eventually leads to a trench growing towards the interior of the island together with the rim [Fig. 4(d)].

For type II islands, the nucleus forms close to the center of the hexagon and initially there is no rim formation [Fig. 4(e)]. Because of the asymmetric corner diffusion known for the growth kinetics on (111) surfaces [8], the nucleus evolves into a dendritic-skeletal shape with three fingers, further on referred to as a dendrite [23]. The dendrite growth during deposition is much slower than the growth of the first-layer area. Accordingly, at the end of the deposition, the island structure is that of a dendrite sitting on top of a first-layer hexagon [Fig. 4(f)].

In the postdeposition regime, the fingers of the dendrite grow very slowly by rare events, where $\mathrm{C}_{60}$ at the island edge overcome the bare dewetting barrier and subsequently attach to the dendrite via diffusion in the second layer. This slow growth continues until one of the three finger tips reaches an island edge. At this moment facilitated dewetting transitions set in and a rim starts to grow along both sides of the finger terminus at the island edge. The formation of the rim is accompanied by a loss of $\mathrm{C}_{60}$ molecules at the rim-free edges causing one of the two other finger tips of the dendrite to reach an island edge. A rim then starts to grow also from this finger terminus and thereafter also the third finger tip reaches the island edge with subsequent rim formation [Fig. 4(g)]. Eventually the three growing rims move towards each other close to edge points located halfway between the tip termini. As for hexagons of type I, funnels are forming at these points and subsequently trenches grow towards the island interior between the fingers of the dendrite. The resulting structure with approximate threefold symmetry shows marked agreement between experiment [Fig. 1(f)] and simulation [Fig. 4(h)]. We also verified the prediction that the trench formation starts in the postdeposition regime by performing a series of measurements with NC-AFM imaging started at various elapsed times after deposition [24].

In conclusion, we developed a model elucidating the fundamental principles of fullerene island formation on weakly interacting, insulating substrates. Based on this model, the formation of both compact double-layer triangular and branched hexagonal islands can be understood. The key to understand their characteristic features is the process of facilitated dewetting. In particular, while a weak molecule-substrate interaction is a prerequisite for dewetting, we unravel that the island shapes are governed by the second-layer occupation rather than by the incommensurability between the structures of molecular islands and the substrate.

This work has been supported by the German Research Foundation (DFG) through the Emmy Noether-program
(KU 1980/1-2 and KU 1980/1-3) and research grant RE 1186/12-1.

*philipp.maass@uni-osnabrueck.de

[1] S. R. Forrest, Nature (London) 428, 911 (2004).

[2] W. Lu and C. M. Lieber, Nature Mater. 6, 841 (2007).

[3] G. M. Whitesides, J.P. Mathias, and C. T. Seto, Science 254, 1312 (1991).

[4] J. V. Barth, Annu. Rev. Phys. Chem. 58, 375 (2007).

[5] F. Rosei, M. Schunack, Y. Naitoh, P. Jiang, A. Gourdon, E. Lægsgaard, I. Stensgaard, C. Joachim, and F. Besenbacher, Prog. Surf. Sci. 71, 95 (2003).

[6] H. Brune, Surf. Sci. Rep. 31, 121 (1998).

[7] J. W. Evans, P. A. Thiel, and M. C. Bartelt, Surf. Sci. Rep. 61, 1 (2006).

[8] T. Michely and J. Krug, Islands, Mounds and Atoms: Patterns and Processes in Crystal Growth Far from Equilibrium (Springer, Berlin, 2004).

[9] S. A. Burke, J. M. Topple, and P. Grütter, J. Phys. Condens. Matter 21, 423101 (2009).

[10] L. Tröger, J. Schütte, F. Ostendorf, A. Kühnle, and M. Reichling, Rev. Sci. Instrum. 80, 063703 (2009).

[11] F. Loske, J. Lübbe, J. Schütte, M. Reichling, and A. Kühnle, Phys. Rev. B 82, 155428 (2010).

[12] S. A. Burke, J. M. Mativetsky, S. Fostner, and P. Grütter, Phys. Rev. B 76, 035419 (2007).

[13] Since the same types of structures are also found on (100) surfaces, it can be expected that the substrate lattice is of minor importance.

[14] G. Ehrlich and F. G. Hudda, J. Chem. Phys. 44, 1039 (1966).

[15] R. L. Schwoebel and E. J. Shipsey, J. Appl. Phys. 37, 3682 (1966).

[16] P. A. Gravil, M. Devel, P. Lambin, X. Bouju, C. Girard, and A. A. Lucas, Phys. Rev. B 53, 1622 (1996).

[17] H. Liu, Z. Lin, L. V. Zhigilei, and P. Reinke, J. Phys. Chem. C 112, 4687 (2008).

[18] L. A. Girifalco and M. Hodak, Phys. Rev. B 65, 125404 (2002).

[19] See supplemental material at http://link.aps.org/ supplemental/10.1103/PhysRevLett.107.016101 for a table of the model parameters.

[20] S. Heinrichs, W. Dieterich, and P. Maass, Phys. Rev. B 75, 085437 (2007).

[21] J. Jacobsen, K. W. Jacobsen, and J. K. Nørskov, Surf. Sci. 359, 37 (1996).

[22] E. Cox, M. Li, P.-W. Chung, C. Ghosh, T. S. Rahman, C. J. Jenks, J. W. Evans, and P. A. Thiel, Phys. Rev. B 71, 115414 (2005).

[23] Details of these dendritic structures (degree of ramification, average branch thickness) depend on the time scales for edge and corner diffusion, see [8], chapter 3. This known behavior was not fitted to the specific situation here at the price of introducing further parameters, which are irrelevant for the new features of rim and trench formation.

[24] See supplemental material at http://link.aps.org/ supplemental/10.1103/PhysRevLett.107.016101 for NCAFM images in the postdeposition regime. 